\title{
Advanced ADC Histogram, Perfusion, and Permeability Metrics Show an Association with Survival and Pseudoprogression in Newly Diagnosed Diffuse Intrinsic Pontine Glioma: A Report from the Pediatric Brain Tumor Consortium
}

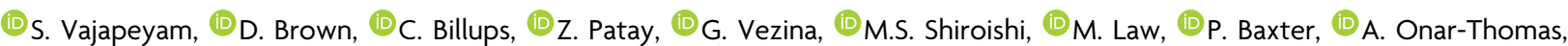
(1) J.R. Fangusaro, (1)I.J. Dunkel, and (10T.Y. Poussaint

\begin{abstract}
BACKGROUND AND PURPOSE: Diffuse intrinsic pontine glioma is a lethal childhood brain cancer with dismal prognosis and MR imaging is the primary methodology used for diagnosis and monitoring. Our aim was to determine whether advanced diffusion, perfusion, and permeability MR imaging metrics predict survival and pseudoprogression in children with newly diagnosed diffuse intrinsic pontine glioma.

MATERIALS AND METHODS: A clinical trial using the poly (adenosine diphosphate ribose) polymerase (PARP) inhibitor veliparib concurrently with radiation therapy, followed by maintenance therapy with veliparib + temozolomide, in children with diffuse intrinsic pontine glioma was conducted by the Pediatric Brain Tumor Consortium. Standard MR imaging, DWI, dynamic contrast-enhanced perfusion, and DSC perfusion were performed at baseline and approximately every 2 months throughout treatment. ADC histogram metrics of T2-weighted FLAIR and enhancing tumor volume, dynamic contrast-enhanced permeability metrics for enhancing tumors, and tumor relative CBV from DSC perfusion MR imaging were calculated. Baseline values, post-radiation therapy changes, and longitudinal trends for all metrics were evaluated for associations with survival and pseudoprogression.
\end{abstract}

RESULTS: Fifty children were evaluable for survival analyses. Higher baseline relative CBV was associated with shorter progression-free survival $(P=.02, Q=0.089)$ and overall survival $(P=.006, Q=0.055)$. Associations of higher baseline mean transfer constant from the blood plasma into the extravascular extracellular space with shorter progression-free survival $(P=.03, Q=0.105)$ and overall survival $(P=.03, Q=0.102)$ trended toward significance. An increase in relative CBV with time was associated with shorter progression-free survival $(P<.001, Q<0.001)$ and overall survival $(P=.004, Q=0.043)$. Associations of longitudinal mean extravascular extracellular volume fraction with progression-free survival $(P=.03, Q=0.104)$ and overall survival $(P=.03, Q=0.105)$ and maximum transfer constant from the blood plasma into the extravascular extracellular space with progression-free survival $(P=.03, Q=0.102)$ trended toward signifcance. Greater increases with time were associated with worse outcomes. True radiologic progression showed greater post-radiation therapy decreases in mode_ADC_FLAIR compared with pseudoprogression (means, -268.15 versus $-26.11, P=.01$.)

CONCLUSIONS: ADC histogram, perfusion, and permeability MR imaging metrics in diffuse intrinsic pontine glioma are useful in predicting survival and pseudoprogression.

ABBREVIATIONS: $D C E$ = dynamic contrast-enhanced; $D I P G=$ diffuse intrinsic pontine glioma; $K_{e p}=$ rate constant from extravascular extracellular space back into blood plasma; $K^{\text {trans }}=$ transfer constant from blood plasma into extravascular extracellular space; OS $=$ overall survival; PBTC $=$ Pediatric Brain Tumor Consortium; PFS = progression-free survival; rCBV = relative CBV; RT = radiation therapy; $T M Z=$ temozolomide; $v_{e}=$ extravascular extracellular volume fraction; $v_{p}=$ blood-plasma volume fraction

Received November 21, 2019; accepted after revision February 10, 2020.

From the Radiology (S.V., T.Y.P.), Boston Children's Hospital and Harvard Medical School, Boston, Massachusetts; DF/HCC Tumor Imaging Metrics Core (D.B.), Massachusetts General Hospital, Boston, Massachusetts; Biostatistics (C.B., A.O.-T.) and Diagnostic Imaging (Z.P.), St. Jude Children's Research Hospital, Memphis, Tennessee; Radiology (G.V.), Children's National Medical Center, Washington, DC; Radiology (M.S.S.), Keck Medical Center of USC, Los Angeles, California; Neuroscience (M.L.), Monash University, Melbourne, Australia; Cancer and Hematology Center (P.B.), Texas Children's Hospital, Houston, Texas; Aflac Cancer and Blood Disorders Center (J.R.F.), Children's Healthcare of Atlanta, Atlanta, Georgia; and Pediatrics (I.J.D.), Memorial Sloan Kettering Cancer Center, New York, New York.
This work was supported by the National Institutes of Health (UM1 CA081457 and P30 CA008748), the Pediatric Brain Tumor Consortium Foundation, the Pediatric Brain Tumor Foundation of the United States, and the American Lebanese Syrian Associated Charities.

Paper previously presented at: Annual Meeting of the American Society of Neuroradiology, May 18-23, 2019; Boston, Massachusetts.

Please address correspondence to Sridhar Vajapeyam, PhD, Boston Children's Hospital and Harvard Medical School, Department of Radiology, 300 Longwood Ave, Boston, MA 02115; e-mail: sridhar.vajapeyam@childrens.harvard.edu

- Indicates open access to non-subscribers at www.ajnr.org

$\equiv$ Indicates article with supplemental on-line tables.

http://dx.doi.org/10.3174/ajnr.A6499 
D iffuseintrinsic pontine gliomas (DIPGs) represent $80 \%$ of all brain stem gliomas, and brain stem tumors constitute approximately $10 \%-15 \%$ of pediatric CNS tumors. ${ }^{1}$ The prognosis for DIPG has been uniformly dismal, with $>90 \%$ of patients succumbing to the disease within 2 years of diagnosis, with a median overall survival of 9 months. ${ }^{2}$ Radiation therapy (RT) has shown transient improvement of neurologic symptoms and marginal survival benefit. $^{3}$

In this study, we assessed associations between survival and imaging metrics derived from advanced diffusion, perfusion, and permeability MR imaging data in a cohort of patients with newly diagnosed DIPG treated with veliparib concurrent with RT, followed by maintenance therapy of veliparib plus temozolomide (TMZ) treated in a Pediatric Brain Tumor Consortium (PBTC) Phase I/II trial. Poly (adenosine diphospate ribose) polymerase (PARP) inhibitors such as veliparib have been shown to overcome TMZ resistance while also increasing radiosensitivity. ${ }^{4,5}$ Advanced ADC histogram metrics have been shown to correlate with survival ${ }^{6}$ in DIPG, whereas perfusion and permeability metrics have shown associations with survival as well as pseudoprogression $^{7-9}$ in pediatric and adult gliomas. We therefore explored the utility of these imaging metrics to predict pseudoprogression versus early true progression.

\section{MATERIALS AND METHODS}

\section{Patients and Treatment Protocol}

Fifty-three children (33 girls, 20 boys; median age 6.6 years; range, 2.5-12.9 years) with newly diagnosed DIPGs were enrolled in a Phase II PBTC trial (PBTC-033, NCT01514201) to determine the efficacy of administering veliparib concurrently with radiation therapy, followed by maintenance therapy with veliparib and temozolomide (TMZ). All patients received photon radiation with a planned target volume of $5400 \mathrm{cGy}$ administered in 30 fractions of $180 \mathrm{cGy}$ and a $50-\mathrm{mg} / \mathrm{m}^{2}$ dose of veliparib, followed by maintenance therapy with veliparib at $25 \mathrm{mg} / \mathrm{m}^{2}$ two times a day and TMZ at $135 \mathrm{mg} / \mathrm{m}^{2} /$ day for 5 days every 28 days.

\section{Imaging and Image Analysis}

MR Imaging Evaluation. Standard MR imaging (which included axial T2, axial T2-weighted FLAIR and axial pre- and postcontrast T1-weighted images) was performed within 2 weeks before registration; at the end of radiation therapy (week 10); within 1 week before courses 3,5 , and 8 of maintenance therapy; and at the end of treatment. Images were transferred to a Vitrea workstation (Vital Images, Minnetonka, Minnesota), and 3D FLAIR and enhancing tumor volumes were calculated from the axial T2weighted FLAIR and axial postcontrast T1-weighted images, respectively. DSC perfusion, dynamic contrast-enhanced (DCE) permeability imaging, and DTI were performed at the same time points as standard MR imaging through week 26 (6 months from the initiation of treatment).

DCE Permeability Imaging and Analysis. All patients underwent a DCE-MR imaging protocol as follows: 1) variable flip angle echo-spoiled gradient-echo T1-mapping sequences using flip angles of $15^{\circ}, 10^{\circ}, 5^{\circ}$, and $2^{\circ}$; $\mathrm{TR}=5$ seconds; $\mathrm{TE}=$ minimum;
FOV $=240 \mathrm{~mm}$; section thickness $=5 \mathrm{~mm} ; 2$ ) A 3D fast gradient-echo DCE-MR imaging axial sequence of 50 phases, 7 seconds apart, with flip angle $=15^{\circ}, \mathrm{TR}=4$ seconds, $\mathrm{TE}=$ minimum. FOV, section thickness, and scan locations were identical to those in the T1-mapping sequences to allow the use of the T1-maps to calculate permeability metrics. A bolus of gadobutrol $(0.05 \mathrm{mmol} / \mathrm{kg}$ of body weight $)$ was injected 20 seconds after the start of scanning.

DCE-MR images were transferred to a DynaCAD workstation (https://www.invivocorp.com/solutions; Invivo, Gainesville, Florida) for automated processing using OmniLook software. Voxelwise T1-maps were generated from the variable flip angle images as described in Fram et $\mathrm{al}^{10}{ }^{10}$ and the 2-compartment extended Tofts model ${ }^{11}$ was used to generate maps of the pharmacokinetic parameters, namely transfer constant from the blood plasma into the extravascular extracellular space $\left(K^{\text {trans }}\right)$, rate constant from extravascular extracellular space back into blood plasma $\left(\mathrm{k}_{\mathrm{ep}}\right)$, extravascular extracellular volume fraction $\left(\mathrm{v}_{\mathrm{e}}\right)$, and blood plasma volume fraction $\left(\mathrm{v}_{\mathrm{p}}\right)$. ROIs were drawn on each section of tumor around contrast-enhancing portions of the tumor by an imaging data analyst or by a $\mathrm{PhD}$ scientist and verified by a Certificate of Added Qualification-certified neuroradiologist, and the mean (over voxels) and SDs of each of the variables were recorded for statistical analysis.

DSC Perfusion Imaging and Analysis. An axial 2D T2* gradient recalled-echo EPI sequence was used with $\mathrm{TR}=2000 \mathrm{~ms}$, $\mathrm{TE}=$ $23 \mathrm{~ms}$, section thickness $=5.0 \mathrm{~mm}$ with a $2-\mathrm{mm}$ gap, flip angle $=$ $60^{\circ}$, and 50-60 time points. With the bolus for the DCE-MR imaging serving as preloading, another bolus injection of $0.05 \mathrm{mmol} / \mathrm{kg}$ body weight of gadobutrol was administered 20 seconds after start of scanning, followed by a $10-\mathrm{mL}$ saline flush. Corrected CBV maps ${ }^{12}$ were generated off-line and normalized using an ROI selected in normal-appearing white matter. A 2D ROI corresponding to the area of greatest enhancement in the tumor was selected in the normalized relative CBV (rCBV) map and used as the rCBV of the tumor.

DTI Acquisition and ADC Histogram Analysis. DTI data were acquired with the following acquisition parameters on a $3 \mathrm{~T}$ scanner: section thickness $=2.2 \mathrm{~mm}, \mathrm{TR}=8800 \mathrm{~ms}$, TE $=88 \mathrm{~ms}$, FOV $=220 \mathrm{~mm}$, b-value $=1000 \mathrm{~s} / \mathrm{mm}^{2}, 35$ directions. Due to their superior anatomic contrast, axial $\mathrm{B}_{0}$ images from the DTI sequence were registered to the axial FLAIR images using the mutual information algorithm in FSL (www.fmrib.ox.ac.uk/ fsl), ${ }^{13,14}$ and the same transformation matrix was used on the ADC maps to register them to the FLAIR images. Axial postcontrast images were also similarly registered to FLAIR images. $3 \mathrm{D}$ ROIs comprising the tumor FLAIR volume and the postcontrast enhancing tumor volume were automatically generated using the thresholding feature in Fiji (https://imagej.net/Fiji/Downloads). ${ }^{15}$ The ADC values of the voxels in FLAIR and enhancing volumes were then used to generate the ADC_FLAIR and ADC_enhancing volumes, respectively. These volumes were thresholded using a uniform range of $600-2600 \times 10^{-6} \mathrm{~mm}^{2} / \mathrm{s}$ to automatically exclude cysts, necrosis, and hemorrhage, and corresponding ADC_FLAIR and ADC_enhancing histograms were generated. Histogram 
metrics used for statistical analysis were the number of peaks (unimodal or bimodal), mean, SD, skewness, and kurtosis of these histograms. In the case of tumors showing bimodal ADC histograms, the lower peak was measured for mean and SD, and skewness and kurtosis were not recorded.

For each of the imaging metrics, the baseline value, the postRT change (defined as the value at the 10 -week post-RT scan minus the baseline value), and the time-dependent longitudinal change in the metric during the course of treatment were examined for associations with progression-free survival (PFS), overall survival (OS), and pseudoprogression.

For patients suspected of having pseudoprogression during the first 6 months of therapy, the treating physician had the option of allowing the patient to continue protocol treatment and repeat the disease assessment in 4-6 weeks. Pseudoprogression was defined as tumors that met the $\geq 25 \%$ progressive disease threshold by bidimensional MR imaging area but improved spontaneously to a size of stable disease or smaller compared with initial imaging on subsequent scans. Provided that the patient did not show clinical deterioration consistent with tumor progression and subsequent MR imaging demonstrated tumor regression or stable disease, the patient could remain on treatment. If the repeat MR imaging after 4-6 weeks showed true radiographic disease progression, rather than pseudoprogression, then the date of progression was the date of the initial MR imaging, not the follow-up scan.

\section{Statistical Methods}

To examine associations between imaging parameters and outcome, we used only eligible and evaluable Phase II patients. OS was defined as the time interval from the date of treatment to the date of death from any cause or to the date of last follow-up. PFS was defined as the time interval from the date of treatment to the earliest date of failure (disease progression or death from any cause) for patients who failed or to the date of last contact for patients who had not failed.

Exact Wilcoxon rank sum tests were used to compare distributions of continuous imaging metrics among patient groups. For each of the imaging metrics, the baseline value, the post-RT change (defined as the value at the 10 -week post-RT scan minus the baseline value), and the time-dependent longitudinal change in the metrics during the course of treatment were examined for associations with outcome (PFS and OS) using Cox regression models. Overall survival was compared among those with pseudoprogression versus those with true early progression using an exact log-rank test. For exploratory analyses examining associations with outcome (OS and PFS), unadjusted $P$ values are reported along with $Q$ values based on the false discovery rate, given the large number of statistical tests performed. These were calculated using the fdrtool package in R (http://www.r-project. org). ${ }^{16,17} Q$ values less than a fixed threshold of 0.1 were considered significant.

\section{RESULTS}

\section{Patients}

Of 53 patients enrolled in the study, 1 patient withdrew before therapy and 2 patients received $<1$ full dose of veliparib, leaving
50 patients evaluable for survival analyses. Forty-three patients died, and 45 experienced a PFS event. Seven patients had suspected pseudoprogression confirmed by repeat imaging and were compared with 20 patients identified as having true progression in the first 6 months of treatment. None of the patients had intratumoral hemorrhage, and no biopsy was performed.

\section{MR Imaging Evaluation}

Most of the tumors were T1-hypointense and T2-hyperintense at presentation. Larger baseline tumor volumes on FLAIR were associated with worse PFS $(P=.01, Q=0.079)$ but not OS. Larger enhancing tumor volumes at baseline were associated with worse $\operatorname{OS}(P=.005, Q=0.047)$ and PFS $(P=.01, Q=0.070)$. Larger percentage increases in FLAIR tumor volume with RT correlated with worse PFS $(P=.01, Q=0.084)$. Larger increases in enhancing tumor volume with RT correlated with worse PFS $(P=.01, Q=0.078)$. Volumetric analysis showed no difference between pseudoprogression and true early progression groups.

\section{DCE Permeability}

Associations of higher baseline mean $K^{\text {trans }}(n=22)$ and shorter OS $(P=.03, Q=0.102)$ and PFS $(P=.03, Q=0.105)$ trended toward significance. When analyzed as continuous time-dependent variables, associations of mean $v_{\mathrm{e}}$ with PFS $(P=.03, Q=0.104)$ and $O S(P=.03, Q=0.105)$ and maximum $K^{\text {trans }}$ with PFS $(P=.03, Q=0.102)$ trended toward significance. Greater increases with time were associated with worse outcomes (Fig 1). Permeability metrics showed no difference between pseudoprogression and true early progression groups.

\section{DSC Perfusion}

Higher baseline rCBV was associated with shorter PFS $(P=.02$, $Q=0.089)$ and $\operatorname{OS}(P=.006, Q=0.055)$. When analyzed as a time-dependent variable, an increase in $\mathrm{rCBV}$ with time was associated with shorter OS $(P=.004, Q=0.043)$ and PFS $(P<.001, Q<0.001)$. Post-RT change in $\mathrm{rCBV}$ was not associated with survival or pseudoprogression.

\section{ADC Histogram Analyses}

Associations of higher baseline mode_ADC_enhancing with longer PFS $(P=.03, Q=0.106)$ and of greater post-RT increase in skewness_ADC_FLAIR with longer PFS $(P=.03, Q=0.102)$ trended toward significance. Mode_ADC_FLAIR showed a greater decrease after RT for true early progression compared with tumors that showed pseudoprogression (means, -268.15 versus $-26.11, P=.01$ ) (Fig 2).

A complete set of results of all relevant statistical tests can be found in the On-line Tables 1-7.

\section{DISCUSSION}

While some early studies concluded that standard imaging could not predict survival in DIPG,${ }^{18}$ several more recent studies have shown metrics derived from standard imaging, such as baseline and post-RT changes on FLAIR, enhancing tumor volume, and the presence or absence of enhancement at baseline, are correlated with survival. ${ }^{19-21}$ Our results showing associations between larger baseline FLAIR and enhancing tumor 


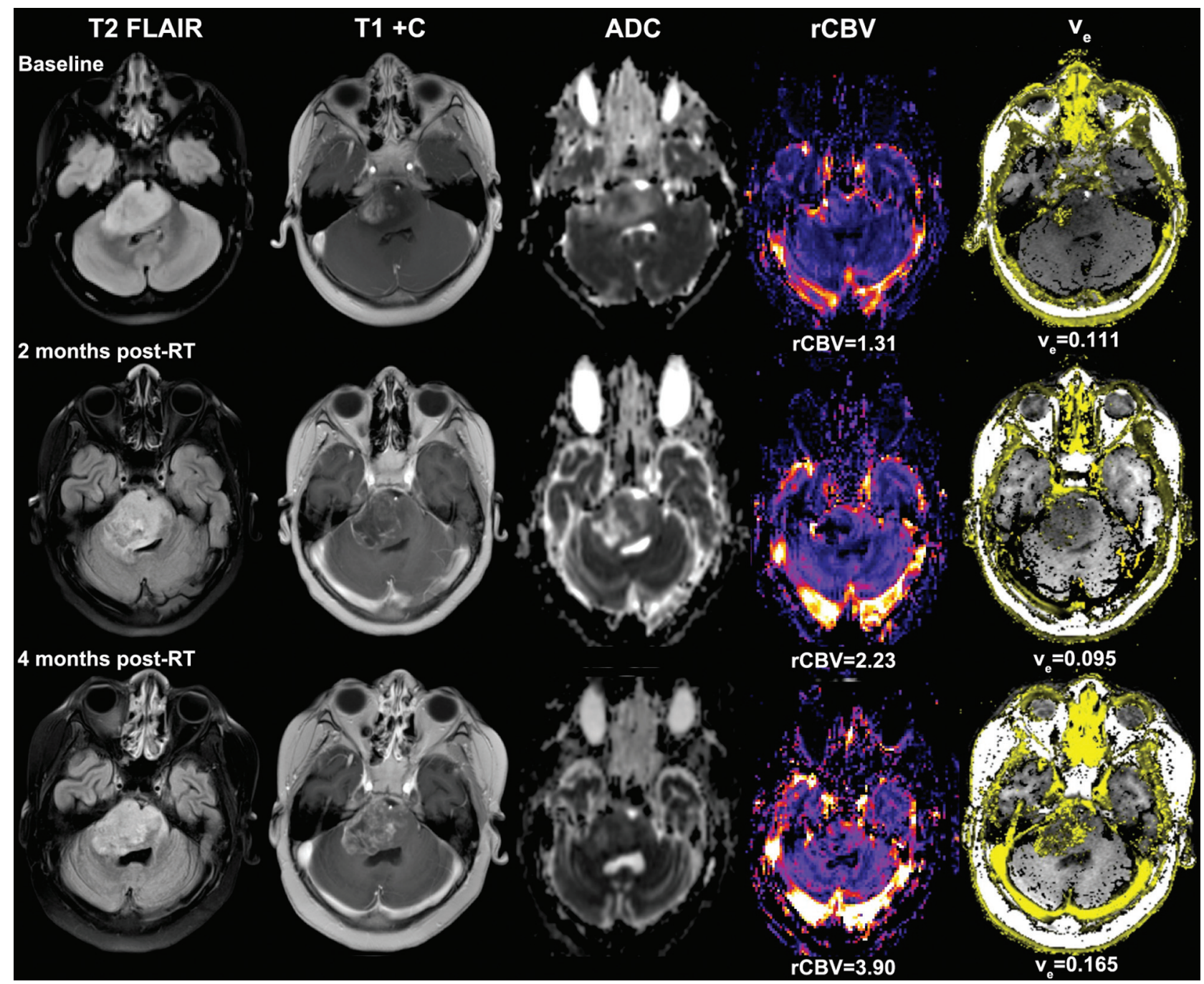

FIG 1. Large increase with time in rCBV and mean $v_{e}$ in a 6.6-year-old female short-term survivor (OS $=124$ days) with DIPG. Increase in $r C B V$ with time was associated with shorter PFS $(P=.004, Q=0.043)$ and $O S(P<.001, Q<0.001)$. An increase in mean $v_{\mathrm{e}}$ was marginally associated with shorter PFS $(P=.028, Q=0.104)$ and $O S(P=.030, Q=0.105)$.

volumes and survival are further confirmation of the role of standard MR imaging in predicting survival.

Advanced MR imaging techniques such as DSC and DCEMR perfusion imaging, ${ }^{22-24}$ arterial spin-labeling, ${ }^{8}$ diffusion imaging including ADC histograms and functional diffusion maps, ${ }^{6,22,25}$ MR spectroscopy, ${ }^{26}$ and $\mathrm{PET}^{27}$ have proved increasingly useful in characterizing the heterogeneity in DIPG for predicting survival and may even provide insight into molecular subgroups. $^{28,29}$

Our DCE-MR imaging findings show that increased baseline mean $K^{\text {trans }}$ was associated with shorter survival, which again suggests that greater perfusion in enhancing tumors indicates a worse prognosis. The association of increased $\mathrm{v}_{\mathrm{e}}$ and shorter survival found in this study is consistent with an earlier report linking higher $\mathrm{v}_{\mathrm{e}}$ to higher grade tumors in a pediatric population. ${ }^{30} \mathrm{~V}_{\mathrm{e}}$ is the extravascular extracellular volume fraction of the tissue and, thus, is dependent on the cellularity as well as the vascularity of the tumor. Because both cellularity and vascularity change with treatment, our findings suggest that the rate of change of $\mathrm{v}_{\mathrm{e}}$ during the course of treatment could be used to monitor the efficacy of treatment: A rapid increase in $\mathrm{v}_{\mathrm{e}}$ may indicate an ineffective treatment regimen.

Our findings of higher baseline rCBV as well as an increase of $\mathrm{rCBV}$ during treatment being associated with shorter survival is in agreement with earlier findings reported by Hipp et $\mathrm{al}^{24}$ as well as other studies showing that enhancement at baseline and across time is associated with a worse prognosis. ${ }^{20,21}$ Sedlacik et al, ${ }^{23}$ however, found that tumors with a higher baseline cerebral blood flow measured by DSC and a higher increase in these metrics through RT had a longer PFS. Similarly, Calmon et $\mathrm{al}^{8}$ found that higher $\mathrm{rCBV}$ measured immediately following RT correlated with longer PFS. Higher baseline rCBV has, however, been linked to increased angiogenesis ${ }^{31}$ and a biopsy-confirmed higher grade in DIPG. ${ }^{32}$ The lack of consistent DSC perfusion findings in DIPG is likely due to differences in acquisition and postprocessing parameters, susceptibility artifacts associated with the location of the tumor in the brain stem, and also the challenges of choosing a representative $2 \mathrm{D}$ tumor ROI, given the tumor 


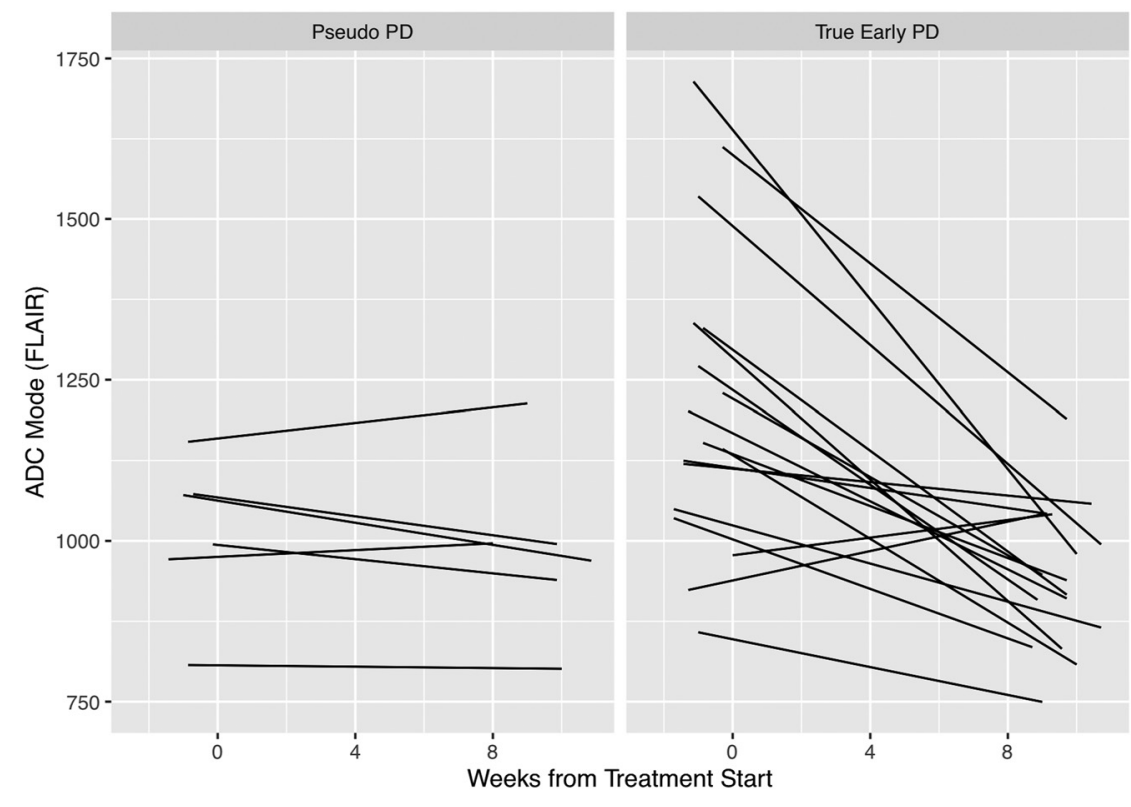

FIG 2. Post-RT change in mode_ADC_FLAIR for pseudoprogression cases on the left and true early progression on the right. The striking difference between groups is evident. Note the sharp drop between baseline and post-RT scans in the mode of ADC_FLAIR for true early progression cases on the right compared with the pseudoprogression cases on the left (means, -268.15 versus -26.11 ; $P=.0099$ ). All ADC values are in $10^{-6} \mathrm{~mm}^{2} / \mathrm{s}$.

heterogeneity seen in DIPG. In addition, it has been shown that there are rapid changes in cerebral blood volume and flow in DIPG both during RT and also immediately following $\mathrm{RT}^{23}$ The time point when DSC perfusion is measured during therapy is therefore critical and may also explain the divergent findings in the literature.

A greater post-RT increase in skewness_ADC_FLAIR implies a larger post-RT drop in ADC and was associated with longer survival in our study, and correlates with published data from a large cohort. ${ }^{6}$ The drop in ADC could be due to a decrease in vasogenic edema, indicating that the radiation therapy is effective. $^{6}$

We report on 7/50 (14\%) cases of pseudoprogression in this DIPG cohort. A post-RT decrease in mode_ADC_FLAIR was significantly lower in those showing pseudoprogression compared with those who went on to show true early progression in our study. While pseudoprogression is well-documented in adult gliblastomas treated with RT + TMZ, typically seen within the first 3 months after radiation, ${ }^{33}$ there are very few reports on DIPG. Chassot et a ${ }^{34}$ reported $4 / 22$ (18\%) cases of pseudoprogression in children with DIPG treated with RT + TMZ and found no difference in overall survival between pseudoprogression and true progression cases. Carceller et al ${ }^{35}$ reported 6/44 (13.6\%) cases of pseudoprogression in a DIPG cohort treated with various different treatment regimens and found no difference in overall survival between pseudoprogression and true progression groups. We also observed no evidence of a significant difference in overall survival among our patients with pseudoprogression versus those with true early progression $(P=.75)$. In a peptide-based vaccine DIPG trial, Ceschin et $\mathrm{al}^{36}$ found that parametric response maps of ADC were able to retrospectively identify 4/21 (19\%) cases of pseudoprogression and reported better overall survival in these cases. More recently, Calmon et $\mathrm{al}^{37}$ reported 19/43 (44\%) cases of pseudoprogression in a DIPG cohort treated with RT along with various concurrent chemotherapy agents in 37 of the cases. There are thus very few cases of pseudoprogression reported in the literature, confounded by the fact that they have all undergone varying treatment regimens. In addition, the definition of pseudoprogression is inconsistent across these reports and affects the frequency of the occurrence of pseudoprogression reported.

While most previous studies have retrospectively compared diffusion and perfusion properties of pseudoprogression and true progression groups at the time of radiologic progression, ${ }^{7,9,36,38,39}$ our results show post-RT changes in ADC histograms that precede radiologic progression and could be used to predict pseudoprogression. A recent report by Calmon et $\mathrm{al}^{37}$ found differences in post-RT changes in DSC perfusion between pseudoprogression and true progression groups, confirming the usefulness of looking at post-RT changes in DIPG as a predictive radiologic marker of pseudoprogression. Because pseudoprogression is most often seen within the first 3 months after RT, it is plausible that radiosensitivity and response to radiation affect the incidence of pseudoprogression.

Our understanding of DIPG has changed in the past few years with the advent of biopsy for DIPG and the subsequent discovery of novel histone and genetic mutations. ${ }^{29}$ This study preceded many of these findings and could benefit from genetic and molecular information available from biopsy data. The methodology outlined in this study, particularly the 3D volumetric ADC histogram analyses, may be particularly well-suited to study these tumors. Future work will focus on correlating these advanced diffusion, perfusion, and permeability metrics with molecular signatures.

\section{CONCLUSIONS}

The data from this study demonstrate that higher baseline FLAIR and enhancing tumor volumes, $\mathrm{rCBV}$, and mean $K^{\text {trans }}$ are all associated with shorter survival in newly diagnosed DIPGs treated with veliparib given concurrently with focal radiation, followed by maintenance therapy of veliparib + TMZ in children with DIPG. Smaller percentage increases in FLAIR tumor volume, smaller increases in enhancing tumor volume, smaller increases in skewness of ADC_FLAIR, and larger decreases of mean_ADC_enhancing were associated with longer survival. Higher increases with time in $\mathrm{rCBV}$ and $\mathrm{V}_{\mathrm{e}}$ in the course of treatment were associated with shorter survival. Tumors with pseudoprogression had a smaller post-RT 
decrease in mode_ADC_FLAIR than those that had true early progressive disease. These results incorporating advanced diffusion, perfusion, and permeability metrics and the association with survival and pseudoprogression will need to be validated in larger prospective clinical trials.

Disclosures: Sridhar Vajapeyam—RELATED: Grant: National Institutes of Health.* Ira J. Dunkel—UNRELATED: Consultancy: Apexigen, Bristol-Myers Squibb, Celgene, Pfizer, Roche, Comments: all unpaid or de minimis compensation; Grants/Grants Pending: Bristol-Myers Squibb, Genentech, Novartis, Comments: Funds were provided to my institution to cover the cost of clinical trials for which I was institutional Principal Investigator.* Tina Young Poussaint-RELATED: Grant: Pediatric Brain Tumor Consortium Neuroimaging Center Grant, Comments: National Institutes of Health subcontract from the Pediatric Brain Tumor Consortium*; UNRELATED: Royalties: Springer, Comments: Atlas of Pediatric Brain Tumors. Patricia Baxter-RELATED: Grant: National Cancer Institute, Comments: Pediatric Brain Tumor Consortium (UOI) (2UM1CA081457)*; Support for Travel to Meetings for the Study or Other Purposes: Pediatric Brain Tumor Consortium (UOI) (2UMICA081457).* Arzu Onar-Thomas-RELATED: Grant: National Cancer Institute Cancer Therapy Evaluation Program Pediatric Brain Tumor Consortium grant (2UMICA081457)*; UNRELATED: Consultancy: Roche, Eli Lilly, Comments: participation on Pediatric Advisory Committee for 1 specific project for each company; Grants/Grants Pending: Pfizer, Celgene, Apexigen, Novartis, Merck, Comments: grants for clinical trial costs conducted by the Pediatric Brain Tumor Consortium.* *Money paid to the institution.

\section{REFERENCES}

1. Ostrom QT, Gittleman H, Truitt G, et al. CBTRUS statistical report: primary brain and other central nervous system tumors diagnosed in the United States in 2011-2015. Neuro Oncol 2018;20:iv1-86 CrossRef Medline

2. Hargrave D, Bartels U, Bouffet E, Diffuse brainstem glioma in children: critical review of clinical trials. Lancet Oncol 2006;7:241-48 CrossRef Medline

3. Gallitto M, Lazarev S, Wasserman I, et al. Role of radiation therapy in the management of diffuse intrinsic pontine glioma: a systematic review. Adv Radiat Oncol 2019;4:520-31 CrossRef Medline

4. Lemasson B, Wang H, Galban S, et al. Evaluation of concurrent radiation, temozolomide and ABT-888 treatment followed by maintenance therapy with temozolomide and ABT- 888 in a genetically engineered glioblastoma mouse model. Neoplasia 2016;18:82-89 CrossRef Medline

5. Yuan AL, Ricks CB, Bohm AK, et al. ABT-888 restores sensitivity in temozolomide resistant glioma cells and xenografts. PLoS One 2018;13:e0202860 CrossRef Medline

6. Poussaint TY, Vajapeyam S, Ricci KI, et al. Apparent diffusion coefficient histogram metrics correlate with survival in diffuse intrinsic pontine glioma: a report from the Pediatric Brain Tumor Consortium. Neuro Oncol 2016;18:725-34 CrossRef Medline

7. Wan B, Wang S, Tu M, et al. The diagnostic performance of perfusion MRI for differentiating glioma recurrence from pseudoprogression: a meta-analysis. Medicine (Baltimore) 2017;96:e6333 CrossRef Medline

8. Calmon R, Puget $\mathrm{S}$, Varlet $\mathrm{P}$, et al. Multimodal magnetic resonance imaging of treatment-induced changes to diffuse infiltrating pontine gliomas in children and correlation to patient progression-free survival. Int J Radiat Oncol Biol Phys 2017;99:476-85 CrossRef Medline

9. Thomas AA, Arevalo-Perez J, Kaley T, et al. Dynamic contrast enhanced T1 MRI perfusion differentiates pseudoprogression from recurrent glioblastoma. J Neurooncol 2015;125:183-90 CrossRef Medline

10. Fram EK, Herfkens RJ, Johnson GA, et al. Rapid calculation of T1 using variable flip angle gradient refocused imaging. Magn Reson Imaging 1987;5:201-08 CrossRef Medline

11. Tofts PS. Modeling tracer kinetics in dynamic Gd-DTPA MR imaging. J Magn Reson Imaging 1997;7:91-101 CrossRef Medline

12. Boxerman JL, Schmainda KM, Weisskoff RM. Relative cerebral blood volume maps corrected for contrast agent extravasation significantly correlate with glioma tumor grade, whereas uncorrected maps do not. AJNR Am J Neuroradiol 2006;27:859-67 Medline
13. Jenkinson $\mathrm{M}$, Bannister $\mathrm{P}$, Brady $\mathrm{M}$, et al. Improved optimization for the robust and accurate linear registration and motion correction of brain images. Neuroimage 2002;17:825-41 CrossRef Medline

14. Jenkinson M, Beckmann CF, Behrens TE, et al. FSL. Neuroimage 2012;62:782-90 CrossRef Medline

15. Schindelin J, Arganda-Carreras I, Frise E, et al. Fiji: an open-source platform for biological-image analysis. Nat Methods 2012;9:676-82 CrossRef Medline

16. Strimmer K. fdrtool: a versatile $\mathbf{R}$ package for estimating local and tail area-based false discovery rates. Bioinformatics 2008;24:1461-62 CrossRef Medline

17. Benjamini Y, Hochberg Y. Controlling the false discovery rate: a practical and powerful approach to multiple testing. Journal of the Royal Statistical Society: Series B (Methodological) 1995;57:289-300 CrossRef

18. Hargrave D, Chuang N, Bouffet E. Conventional MRI cannot predict survival in childhood diffuse intrinsic pontine glioma. $J$ Neurooncol 2008;86:313-19 CrossRef Medline

19. Kilburn LB, Kocak M, Baxter P, et al. A Pediatric Brain Tumor Consortium phase II trial of capecitabine rapidly disintegrating tablets with concomitant radiation therapy in children with newly diagnosed diffuse intrinsic pontine gliomas. Pediatr Blood Cancer 2018;65 CrossRef Medline

20. Poussaint TY, Kocak M, Vajapeyam S, et al. MRI as a central component of clinical trials analysis in brainstem glioma: a report from the Pediatric Brain Tumor Consortium (PBTC). Neuro Oncol 2011;13:417-27 CrossRef Medline

21. Jansen MH, Veldhuijzen van Zanten SE, Sanchez Aliaga E, et al. Survival prediction model of children with diffuse intrinsic pontine glioma based on clinical and radiological criteria. Neuro Oncol 2015;17:160-66 CrossRef Medline

22. Lobel U, Sedlacik J, Reddick WE, et al. Quantitative diffusionweighted and dynamic susceptibility-weighted contrast-enhanced perfusion MR imaging analysis of T2 hypointense lesion components in pediatric diffuse intrinsic pontine glioma. AJNR Am J Neuroradiol 2011;32:315-22 CrossRef Medline

23. Sedlacik J, Winchell A, Kocak M, et al. MR imaging assessment of tumor perfusion and 3D segmented volume at baseline, during treatment, and at tumor progression in children with newly diagnosed diffuse intrinsic pontine glioma. AJNR Am J Neuroradiol 2013;34:1450-55 CrossRef Medline

24. Hipp SJ, Steffen-Smith E, Hammoud D, et al. Predicting outcome of children with diffuse intrinsic pontine gliomas using multiparametric imaging. Neuro Oncol 2011;13:904-09 CrossRef Medline

25. Ceschin R, Kocak M, Vajapeyam S, et al. Quantifying radiation therapy response using apparent diffusion coefficient (ADC) parametric mapping of pediatric diffuse intrinsic pontine glioma: a report from the Pediatric Brain Tumor Consortium. J Neurooncol 2019;143:79-86 CrossRef Medline

26. Lobel U, Hwang S, Edwards A, et al. Discrepant longitudinal volumetric and metabolic evolution of diffuse intrinsic pontine gliomas during treatment: implications for current response assessment strategies. Neuroradiology 2016;58:1027-34 CrossRef Medline

27. Zukotynski K, Vajapeyam S, Fahey FH, et al. Correlation of 18F-FDG PET and MR apparent diffusion coefficient (ADC) histogram metrics with survival in diffuse intrinsic pontine glioma: a report from the pediatric brain tumor consortium. J Nucl Med 2017;58:1264-69 CrossRef Medline

28. Lober RM, Cho YJ, Tang Y, et al. Diffusion-weighted MRI derived apparent diffusion coefficient identifies prognostically distinct subgroups of pediatric diffuse intrinsic pontine glioma. J Neurooncol 2014;117:175-82 CrossRef Medline

29. Castel D, Philippe C, Calmon R, et al. Histone H3F3A and HIST1H3B K27M mutations define two subgroups of diffuse intrinsic pontine gliomas with different prognosis and phenotypes. Acta Neuropathol 2015;130:815-27 CrossRef Medline

30. Vajapeyam S, Stamoulis C, Ricci K, et al. Automated processing of dynamic contrast-enhanced MRI: correlation of advanced 
pharmacokinetic metrics with tumor grade in pediatric brain tumors. AJNR Am J Neuroradiol 2017;38:170-75 CrossRef Medline

31. Conway AE, Reddick WE, Li Y, et al. "Occult” post-contrast signal enhancement in pediatric diffuse intrinsic pontine glioma is the MRI marker of angiogenesis? Neuroradiology 2014;56:405-12 CrossRef Medline

32. Kilday JP, Pringle C, Kamaly-Asl ID, et al. RADI-15: perfusion imaging in presumed pediatric diffuse intrinsic pontine glioma: a UK regional analysis. Neuro Oncol 2018;20(Suppl 2):i172-73 CrossRef

33. Zikou A, Sioka C, Alexiou GA, et al. Radiation necrosis, pseudoprogression, pseudoresponse, and tumor recurrence: imaging challenges for the evaluation of treated gliomas. Contrast Media Mol Imaging 2018;2018:6828396 CrossRef Medline

34. Chassot A, Canale S, Varlet P, et al. Radiotherapy with concurrent and adjuvant temozolomide in children with newly diagnosed diffuse intrinsic pontine glioma. J Neurooncol 2012;106:399-407 CrossRef Medline

35. Carceller F, Fowkes LA, Khabra K, et al. Pseudoprogression in children, adolescents and young adults with non-brainstem high grade glioma and diffuse intrinsic pontine glioma. J Neurooncol 2016;129:109-21 CrossRef Medline

36. Ceschin R, Kurland BF, Abberbock SR, et al. Parametric response mapping of apparent diffusion coefficient as an imaging biomarker to distinguish pseudoprogression from true tumor progression in peptide-based vaccine therapy for pediatric diffuse intrinsic pontine glioma. AJNR Am J Neuroradiol 2015;36:2170-76 CrossRef Medline

37. Calmon R, Puget $S$, Varlet $P$, et al. Cerebral blood flow changes after radiation therapy identifies pseudoprogression in diffuse intrinsic pontine gliomas. Neuro Oncol 2018;20:994-1002 CrossRef Medline

38. Carceller F, Jerome NP, Fowkes LA, et al. Post-radiotherapy apparent diffusion coefficient (ADC) in children and young adults with high-grade gliomas and diffuse intrinsic pontine gliomas. Pediatr Hematol Oncol 2019;36:103-12 CrossRef Medline

39. Song YS, Choi SH, Park CK, et al. True progression versus pseudoprogression in the treatment of glioblastomas: a comparison study of normalized cerebral blood volume and apparent diffusion coefficient by histogram analysis. Korean J Radiol 2013;14:662-72 CrossRef Medline 